Heinz-Dieter Pohl*

Universität Klagenfurt

DOI: $10.4312 /$ linguistica.60.2.381-383

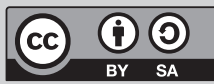

Brenner, Koloman (2018)

\title{
DEUTSCHE MINDERHEIT(EN) UND INSTITUTIONEN. BEITRÄGE ZUR MINDERHEITENKUNDE FÜR GERMANISTIKSTUDENTEN DER FACHRICHTUNG DEUTSCH ALS NATIONALITÄTENSPRACHE.
}

In: Ungarndeutsches Archiv 16, Schriften zur Sprache, Literatur, Kultur und Geschichte der Deutschen in Ungarn. Budapest: ELTE Germanistisches Institut.

194 S. ISSN 1418-1959; ISBN 978-963-284-972-0

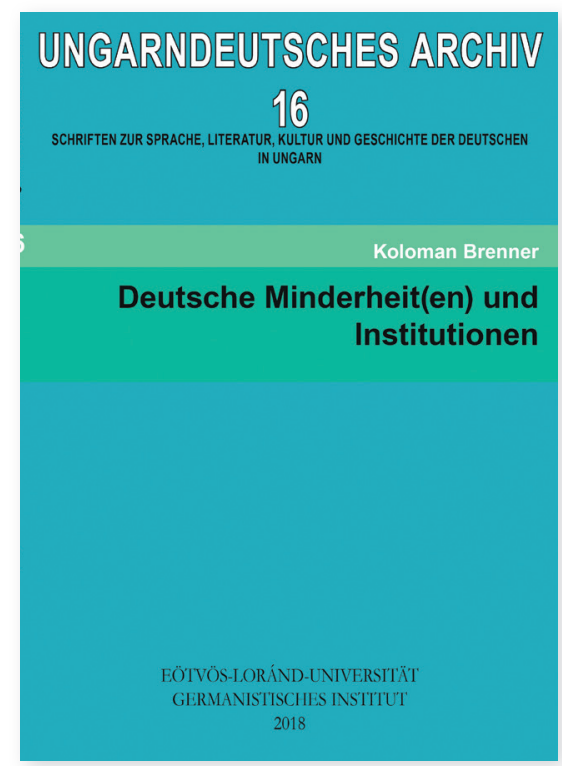

Dieses umfang- und inhaltsreiche Buch beruht zunächst auf der Bearbeitung von Dokumenten zur Sprach- und Minderheitenpolitik in Ungarn nach der Wende von 1989/1990. Dazu kommt dann die Erfahrung aus der langjährigen Expertentätigkeit des Verfassers für die Europäische Charta der Regional- oder Minderheitensprachen, wobei diesem Thema auch die Erfahrungen, Besuche und Studien durch die Vermittlung des Netzwerks der deutschen Minderheiten im Dachverband der Föderalistischen Union Europäischer Nationalitäten (FUEN), der Arbeitsgemeinschaft Deutscher Minderheiten

*heinz.pohl@chello.at 
(https://agdm.fuen.org) zu Gute kommen, ist doch der Verfasser in diesem Bereich seit über 20 Jahren tätig. So können viele Prozesse und Entscheidungen in und über die deutschen Minderheiten in Europa aus der Perspektive eines Insiders dargestellt werden sowie tiefe Einblicke zur Lage der deutschen Minderheiten geboten werden.

Wie der Verfasser im Vorwort ausführt, ergibt sich die Aktualität dieses Buches aus der Tatsache, dass in letzter Zeit auch in der Bundesrepublik Deutschland das Thema „Deutsche Minderheiten“ auf höchster politischen Ebene großes Interesse gefunden hat - nicht zuletzt durch die Tätigkeit der Beauftragten der Bundesregierung für Aussiedler und nationale Minderheiten. Gleichzeitig wuchs das Interesse für die Geschichte und Gegenwart der deutschen Minderheiten in den betroffenen Ländern Ostmitteleuropas. Heute liegt auch ein offizielles Informationsmaterial mit dem Titel „Deutsche Minderheiten stellen sich vor" (Broschüre, Berlin 1916, hg. vom Bundesministerium des Innern) als Teil der Öffentlichkeitsarbeit der deutschen Bundesregierung vor.

Im neuen Bachelor-, Master- und Lehrerausbildungsstudium der Fachrichtung Deutsch als Minderheitensprache am Germanistischen Institut der Universität Budapest sind Minderheitenrechte und Institutionen auch als Lehrveranstaltung vorgesehen. Somit steht mit dem vorliegenden Buch ein Werk zur Verfügung, das den neuesten Stand der Forschung zu den deutschen Minderheiten in Europa wiedergibt, nicht nur in Ungarn, sondern europaweit.

Der Aufbau des Buches ist sehr übersichtlich, der Teil I erläutert die Grundbegriffe und bietet eine Einführung in das komplexe Thema. Der Begriff der „Nation“ sei ein heftig diskutierter und je nach ideologischem Muster unterschiedlich ausgelegter Begriff, was auch heute noch im 21. Jahrhundert in den aktuellen Diskussionen zu beobachten ist. Der moderne europäische Nationsbegriff entstand erst durch die z. T. divergierenden politisch-ideologisch motivierten Entwicklungen auf unserem Kontinent nach der französischen Revolution. Die Nation selbst sei eine Idee, eine abstrakte Bezeichnung, historisch gesehen eigentlich eine konstruierte Identität. Daher ist es notwendig, die wichtigen Grundbegriffe wie „Nation“, „Nationalität“ und „Minderheit“ aus unterschiedlichen wissenschaftlichen Perspektiven und theoretischen Grundlagen entsprechend darzustellen. Da die Sprache ein zentrales Standbein der ethnischen bzw. nationalen Identität ist, wird auch der Zusammenhang zwischen Sprache und Identität ausführlich erläutert, wobei ich anerkennend feststellen kann, dass es dem Verfasser bestens gelungen ist, hier eine objektive Darstellung zu dieser Thematik vorzulegen.

Der Teil II ist schließlich dem Hauptthema „Deutsche Minderheit(en) - Autonomien und Institutionen“ gewidmet, beginnend mit „Deutsche Minderheiten in Ost-Mittel-Europa nach der politischen Wende 1989/1990“. Mit einem Rückblick auf die Zeit zuvor, denn bis kurz vor der Wendezeit wurde die Vertreibung der Deutschen aus dem östlichen und mittleren Teil Europas in den betroffenen Staaten weitgehend tabuisiert und stand auch in den deutschsprachigen Ländern nur am Rande auf der Tagesordnung. Dies hat sich dann seit der Jahrtausendwende verändert und im geschlossenen deutschen Sprachraum wird immer objektiver und detaillierter auch über das Schicksal der Deutschen aus dem Osten berichtet. Die einstige Verbreitung der deutschen Minderheiten zeigt eine Karte des deutschen Sprachgebietes in der Zwischenkriegszeit (S. 42). 
Dazu auch einige Hinweise zu den Eigenbezeichnungen wie „Siebenbürger Sachsen, Donauschwaben, Ungarndeutsche“.

Die einzelnen Staaten: Slowenien (ca. 3.000-5.000), Tschechische Republik (VZ 2011: 18.700), Slowakei (VZ 2011: 4690), Kroatien (ca. 3.000), Serbien (VZ 2011: 4.064), Rumänien (VZ 2011: 40.000), Polen (lt. VZ 150.000, man schätzt aber weit mehr als 180.000). Italien wird mit dem „Sonderfall“ Südtirol vorgestellt (insbes. die entscheidenden Faktoren, die zur Herausbildung der als mustergültig geltenden territorialen Autonomie in Südtirol geführt haben).

Ungarn ist ein eigenes Kapitel gewidmet: „Die deutsche Minderheit in Ungarn im 19.-20. Jahrhundert aus der Perspektive des Minderheitenschutzes“. Einleitend (S. 57) stellt der Verfasser fest: Im heutigen Ungarn gibt es drei größere Siedlungsgebiete, wo Angehörige der deutschen Minderheit in höherer Anzahl leben: Westungarn entlang der österreichischen Grenze, das Ungarische Mittelgebirge (vom Ofner Bergland bis zum Plattensee-Oberland) mit den Zentren Ofen/Buda und Zirc, bzw. Südungarn mit dem Zentrum Fünfkirchen/Pécs. Die Vorfahren der deutschen Minderheit in Westungarn sind „Urbewohner“ dieser Gegend und bildeten ab dem 13.-14. Jahrhundert in wichtigen Zentren wie Ödenburg/Sopron und Wieselburg/Moson die Mehrheitsbevölkerung im ehemaligen Deutsch-Westungarn als Teil des Ungarischen Königreiches (mit einigen Karten). Die Zeit nach 1945 war für die Ungarndeutschen eine schwierige (Enteignung, Diskriminierung usw., teilweise „Aussiedlung“). Den sogenannten „schweren Jahrzehnten“ (1950/60/70er Jahre) folgte etwa seit Mitte der 1980er Jahre die neue Phase einer positiven Entwicklung, z. B. bilinguale Schulen (S. 77). Statistisch stieg die Zahl der ungarischen Staatsbürger, die als Nationalität Deutsch angegeben haben, im Jahre 2011 auf beachtliche 132.000 Personen.

Im Kapitel „Aktuelle Lage der deutschen Minderheit in Ungarn“ werden die heutigen positiven Verhältnisse (Kulturleben, deutsche Sprache im Bildungswesen u.dgl.) dargestellt, zusammen mit deren sprachlich-kulturellen Rahmenbedingungen seit 1989/1990 und dem Ausbau der kulturellen Autonomie der Ungarndeutschen Institutionen. Abschließend berichtet der Autor über den Minderheitenschutz in Europa - Ergebnisse und Perspektiven - und wirft mit dem Abschnitt „Perspektiven im 21. Jahrhundert über den Minderheitenschutz" auch einen Blick in die Zukunft.

Im Anhang findet der Leser die „Europäische Charta der Regional- oder Minderheitensprachen“, das „Rahmenübereinkommen zum Schutz nationaler Minderheiten“, „Aus der Sicht des Minderheitenschutzes völkerrechtlich relevante juristische Dokumente und Beschlüsse seit 1945“ sowie „Ungarndeutsche Institutionen“.

Koloman Brenner hat mit diesem Werk das wohl umfassendste Buch über die deutschen Minderheiten verfasst und darüber hinaus auch ein Handbuch über Minderheiten im allgemeinen vorgelegt, wobei er seine langjährigen Erfahrungen einbringen konnte. 